\title{
A General Hilbert Space Approach to Wavelets and Its Application in Geopotential Determination
}

\author{
Willi Freeden, Oliver Glockner, Rolf Litzenberger
}

\begin{abstract}
A general approach to wavelets is presented within a framework of a separable functional Hilbert space $\mathcal{H}$. Basic tool is the construction of $\mathcal{H}$-product kernels by use of Fourier analysis with respect to an orthonormal basis in $\mathcal{H}$. Scaling function and wavelet are defined in terms of $\mathcal{H}$-product kernels. Wavelets are shown to be 'building blocks' that decorrelate the data. A pyramid scheme provides fast computation. Finally, the determination of the earth's gravitational potential from single and multipole expressions is organized as an example of wavelet approximation in Hilbert space structure.
\end{abstract}

\section{Introduction}

Wavelets are "building blocks" that enable fast decorrelation of data. In other words, three features are incorporated in this way of thinking about wavelets, namely basis property, decorrelation, and fast computation. In the first part of the paper we discuss these aspects in a general (functional) Hilbert space setup. The definition of scaling function and wavelet is based on the concept of product kernels in functional Hilbert spaces. By virtue of the basis property each member of the Hilbert space can be expressed in stable way as linear combination of dilated and shifted copies of a "mother function", i.e. a member of the Hilbert space with vanishing zeroth moment. The wavelet transform maps members of the Hilbert space into an associated two-parameter class of space and scale dependent elements. Wavelets show the power of decorrelation. As a consequence the representation of the data in terms of wavelets is somehow "more compact" than the original representation, that is to say, we search for an accurate approximation by only using a small fraction of the original information of an element of the Hilbert space. Typically the decorrelation is achieved by building wavelets which decay towards low and high frequencies, i.e. in information theory jargon by bandpass filtering. Finally, the main question in wavelet approximation is how to decompose a function into wavelet coefficients, and how to reconstruct efficiently the function under 
consideration from the wavelet coefficients. There is a pyramid algorithm that makes these steps simple and fast. The fast decorrelation power of wavelets is the key to applications such as data compression, fast data transmission, noise cancellation, signal recovering etc. The paper ends with geopotential determination by harmonic wavelets. In this case the product kernels are written as series expansions in terms of single or multipoles. As numerical example we present the wavelet decomposition and reconstruction of the gravitational potential of the earth. The calculations are based on the NASA GSFC and NIMA Joint Geopotential model EGM96 which gives a series expansion of the earth's gravitational potential in terms of multipoles (i.e. solid spherical harmonics).

\section{Basic Settings}

Let $\left(\mathcal{H},(\cdot, \cdot)_{\mathcal{H}}\right)$ be a separable real functional Hilbert space over a certain domain $\Sigma \subset \mathbb{R}^{n}$, i.e. $\mathcal{H}$ consists of functions $F: \Sigma \rightarrow \mathbb{R}$. Furthermore, let $\left\{U_{n}^{*}\right\}_{n=0,1, \ldots}$ be a complete orthonormal system in $\left(\mathcal{H},(\cdot, \cdot)_{\mathcal{H}}\right)$. In a separable real functional Hilbert space $\left(\mathcal{H},(\cdot, \cdot)_{\mathcal{H}}\right)$ any function $F \in \mathcal{H}$ can be represented as Fourier expansion relative to $\left\{U_{n}^{*}\right\}_{n=0,1, \ldots}$ by

$$
F=\sum_{n=0}^{\infty} F^{\wedge}(n) U_{n}^{*}
$$

with "Fourier coefficients"

$$
F^{\wedge}(n)=\left(F, U_{n}^{*}\right)_{\mathcal{H}} \quad, n=0,1,2, \ldots
$$

The idea we follow in this paper is to present in the $\mathcal{H}$-framework a J-level approximation of a function (signal) $F \in \mathcal{H}$ by means of a wavelet analysis. Essential tools are the concept of $\mathcal{H}$-product-kernels and $\mathcal{H}$-convolutions which should be discussed now.

\subsection{Product Kernels}

Any function $\Gamma: \Sigma \times \Sigma \rightarrow \mathbb{R}$ of the form

$$
\Gamma(x, y)=\sum_{n=0}^{\infty} \Gamma^{\wedge}(n) U_{n}^{*}(x) U_{n}^{*}(y),
$$


$x, y \in \Sigma$ with $\Gamma^{\wedge}(n) \in \mathbb{R}, n \in \mathbb{N}_{0}$, is called an $\mathcal{H}$-product kernel (briefly called $\mathcal{H}$-kernel). The sequence $\left\{\Gamma^{\wedge}(n)\right\}_{n=0,1, \ldots}$ is said to be the symbol of the $\mathcal{H}$-kernel (3).

Definition 1.1. A symbol $\left\{\Gamma^{\wedge}(n)\right\}_{n=0,1, \ldots}$ of an $\mathcal{H}$-product kernel (3) is called $\mathcal{H}$-admissible if it satisfies the following conditions:

$$
\begin{aligned}
& \text { (i) } \sum_{n=0}^{\infty}\left(\Gamma^{\wedge}(n)\right)^{2}<\infty, \\
& \text { (ii) } \sum_{n=0}^{\infty}\left(\Gamma^{\wedge}(n) U_{n}^{*}(x)\right)^{2}<\infty
\end{aligned}
$$

for all $x \in \Sigma$.

$\mathcal{H}$-convolutions will be introduced in the following way.

Definition 1.2. Let $F$ be of class $\mathcal{H}$. Suppose that $\Gamma$ is an $\mathcal{H}$-kernel of the form (3) with $\mathcal{H}$-admissible symbol $\left\{\Gamma^{\wedge}(n)\right\}_{n=0,1, \ldots}$, then the convolution of $\Gamma$ against $F$ is defined by

$$
\left(\Gamma *_{\mathcal{H}} F\right)(x)=(\Gamma(x, \cdot), F)_{\mathcal{H}}=\sum_{n=0}^{\infty} \Gamma^{\wedge}(n) F^{\wedge}(n) U_{n}^{*}(x) .
$$

From (4) we immediately see that

$$
\left(\Gamma *_{\mathcal{H}} F\right)^{\wedge}(n)=\Gamma^{\wedge}(n) F^{\wedge}(n), \quad n \in \mathbb{N}_{0} .
$$

The convolution of two $\mathcal{H}$-product kernels with $\mathcal{H}$-admissible symbols leads to the following result.

Theorem 1.3. Let $\Gamma_{1}$ and $\Gamma_{2}$ be $\mathcal{H}$-kernels with $\mathcal{H}$-admissible symbols $\left\{\Gamma_{1}^{\wedge}(n)\right\}_{n=0,1, \ldots}$ and $\left\{\Gamma_{2}^{\wedge}(n)\right\}_{n=0,1, \ldots}$, respectively. Then

$$
\begin{aligned}
\left(\Gamma_{1} * \mathcal{H} \Gamma_{2}\right)(x, y) & =\left(\Gamma_{1} * \mathcal{H} \Gamma_{2}(\cdot, y)\right)(x) \\
& =\left(\Gamma_{1}(x, \cdot), \Gamma_{2}(\cdot, y)\right)_{\mathcal{H}} \\
& =\sum_{n=0}^{\infty} \Gamma_{1}^{\wedge}(n) \Gamma_{2}^{\wedge}(n) U_{n}^{*}(x) U_{n}^{*}(y)
\end{aligned}
$$

holds for all $x, y \in \Sigma$, and $\left\{\left(\Gamma_{1} *_{\mathcal{H}} \Gamma_{2}\right)^{\wedge}(n)\right\}_{n=0,1, \ldots}$ given by

$$
\left(\Gamma_{1} *_{\mathcal{H}} \Gamma_{2}\right)^{\wedge}(n)=\Gamma_{1}^{\wedge}(n) \Gamma_{2}^{\wedge}(n) .
$$

constitutes an $\mathcal{H}$-admissible symbol of the $\mathcal{H}$-kernel $\Gamma_{1} *_{\mathcal{H}} \Gamma_{2}$. 


\section{$1.2 \quad \mathcal{H}$-scaling Functions}

After having explained the convolution between two $\mathcal{H}$-kernels with $\mathcal{H}$-admissible symbols we are now interested in developing countable families $\left\{\Gamma_{J}\right\}, J \in \mathbb{Z}$, of $\mathcal{H}$-product kernels $\Gamma_{J}$ which may be understood as scaling functions in our $\mathcal{H}$-wavelet concept.

As preparation we introduce a dilation operator acting on these families in the following way: Let $\Gamma_{J}$ be a member of the family of product kernels. Then the dilation operator $D_{k}, k \in \mathbb{Z}$, is defined by $D_{k} \Gamma_{J}=\Gamma_{J+k}$. In particular, we have $\Gamma_{J}=D_{J} \Gamma_{0}$. Thus we refer to $\Gamma_{0}$ as the "mother kernel". Moreover, we define a shifting operator $S_{x}, x \in \Sigma, J \in \mathbb{Z}$, by $S_{x} \Gamma_{J}=\Gamma_{J}(x, \cdot)$ and $S_{x} \Gamma_{J}=$ $\Gamma_{J}(\cdot, x)$, respectively. In doing so we consequently get by composition of the operator $\Gamma_{J}(x, \cdot)=S_{x} D_{J} \Gamma_{0}$ for all $x \in \Sigma$ and all $J \in \mathbb{Z}$. Note that all kernels $\Gamma_{J}$ are symmetric, so that $\Gamma_{J}(x, y)=\Gamma_{J}(y, x), x, y \in \Sigma$ for all $J \in \mathbb{Z}$.

We are now in position to introduce scaling functions.

Definition 1.4. Let $\left\{\left(\Phi_{J}\right)^{\wedge}(n)\right\}_{n=0,1, \ldots}, J \in \mathbb{Z}$, define an $\mathcal{H}$-admissible symbol of a family of $\mathcal{H}$-kernels satisfying additionally the following properties:

$$
\begin{aligned}
& \text { (i) } \lim _{J \rightarrow \infty}\left(\left(\Phi_{J}\right)^{\wedge}(n)\right)^{2}=1, \quad n \in \mathbb{N}, \\
& \text { (ii) } \quad\left(\left(\Phi_{J}\right)^{\wedge}(n)\right)^{2} \geq\left(\left(\Phi_{J-1}\right)^{\wedge}(n)\right)^{2}, \quad J \in \mathbb{Z}, n \in \mathbb{N}, \\
& \text { (iii) } \quad \lim _{J \rightarrow-\infty}\left(\left(\Phi_{J}\right)^{\wedge}(n)\right)^{2}=0, \quad n \in \mathbb{N}, \\
& \text { (iv) } \quad\left(\left(\Phi_{J}\right)^{\wedge}(0)\right)^{2}=1, \quad J \in \mathbb{Z} .
\end{aligned}
$$

Then $\left\{\left(\Phi_{J}\right)^{\wedge}(n)\right\}_{n=0,1, \ldots}$ is called the generating symbol of an $\mathcal{H}$-scaling function. The family of $\mathcal{H}$-kernels $\left\{\Phi_{J}\right\}, J \in \mathbb{Z}$, given by

$$
\Phi_{J}(x, y)=\sum_{n=0}^{\infty}\left(\Phi_{J}\right)^{\wedge}(n) U_{n}^{*}(x) U_{n}^{*}(y), \quad x, y \in \Sigma,
$$

is called $\mathcal{H}$-scaling function.

From the results of the previous section it follows immediately that $\Phi_{J}(x, \cdot) \in$ $\mathcal{H}, x \in \Sigma, J \in \mathbb{Z}$. Furthermore it is easily seen that $\Phi_{J} *_{\mathcal{H}} \Phi_{J}$ is an $\mathcal{H}$-kernel with $\mathcal{H}$-admissible symbol $\left\{\left(\left(\Phi_{J}\right)^{\wedge}(n)\right)^{2}\right\}, n=0,1, \ldots$.

This leads us to the following result. 
Theorem 1.5. Let $\left\{\left(\Phi_{J}\right)^{\wedge}(n)\right\}_{n=0,1, \ldots}, J \in \mathbb{Z}$, be the generating symbol of a scaling function $\Phi_{J}$. Then

$$
\lim _{J \rightarrow \infty}\left\|F_{J}-F\right\|_{\mathcal{H}}=0
$$

holds for all $F \in \mathcal{H}$, where $F_{J}$ given by

$$
F_{J}=\left(\Phi_{J} *_{\mathcal{H}} \Phi_{J}\right) *_{\mathcal{H}} F, \quad F \in \mathcal{H}
$$

is said to be the J-level approximation of $F$.

Proof. We introduce the operator $T_{J}: \mathcal{H} \rightarrow \mathcal{H}, J \in \mathbb{Z}$, by

$$
F_{J}=T_{J} F=\left(\Phi_{J} *_{\mathcal{H}} \Phi_{J}\right) *_{\mathcal{H}} F .
$$

From the definition of the convolution and the fact that $\Phi_{J} *_{\mathcal{H}} \Phi_{J}$ is an $\mathcal{H}$-kernel with $\mathcal{H}$-admissible symbol it follows that

$$
T_{J} F=\sum_{n=0}^{\infty}\left(\left(\Phi_{J}\right)^{\wedge}(n)\right)^{2} F^{\wedge}(n) U_{n}^{*} .
$$

But this implies that

$$
\begin{aligned}
\left\|T_{J}\right\| & =\sup _{\substack{G \in \mathcal{H} \\
\|G\|_{\mathcal{H}}=1}}\left\|T_{J} G\right\|_{\mathcal{H}} \\
& \left.=\left(\sum_{n=0}^{\infty}\left(\left(\Phi_{J}\right)^{\wedge}(n)\right)^{4}\left(G^{\wedge}(n)\right)^{2}\right)\right)^{\frac{1}{2}} \\
& \leq \sup _{n \in \mathbb{N}_{0}}\left(\left(\Phi_{J}\right)^{\wedge}(n)\right)^{2}\left(\sum_{n=0}^{\infty}\left(G^{\wedge}(n)\right)^{2}\right)^{\frac{1}{2}} \\
& \leq \sup _{n \in \mathbb{N}_{0}}\left(\left(\Phi_{J}\right)^{\wedge}(n)\right)^{2}<\infty
\end{aligned}
$$

for every $J \in \mathbb{Z}$, since $\left\{\left(\Phi_{J}\right)^{\wedge}(n)\right\}_{n=0,1, \ldots}$ is $\mathcal{H}$-admissible.

Now, from Parseval's identity, we obtain

$$
\begin{aligned}
& \lim _{J \rightarrow \infty}\left\|T_{J} F-F\right\|_{\mathcal{H}}^{2} \\
& =\lim _{J \rightarrow \infty} \sum_{n=0}^{\infty}\left(1-\left(\left(\Phi_{J}\right)^{\wedge}(n)\right)^{2}\right)^{2}\left(F^{\wedge}(n)\right)^{2} .
\end{aligned}
$$


From the conditions (i), (ii) and (iv) of Definition 1.4 we are able to deduce that $\left(\left(\Phi_{J}\right)^{\wedge}(n)\right)^{2} \leq 1$ for $n \in \mathbb{N}_{0}$. But this shows us that

$$
0 \leq\left(1-\left(\left(\Phi_{J}\right)^{\wedge}(n)\right)^{2}\right)^{2} \leq 1
$$

is valid for all $n \in \mathbb{N}_{0}$. Therefore the limit and the infinite sum in (14) may be interchanged. By applying (i) and (iv) we finally arrive at the desired result.

Note that condition (iii) has not been used yet. This condition, however, is needed as assumption for defining $\mathcal{H}$-wavelets and establishing a multiresolution analysis.

According to our construction, for any $F \in \mathcal{H}$, each $T_{J} F$ defined by (11) provides an approximation of $F$ at scale $J$. In terms of filtering $\Phi_{J} *_{\mathcal{H}} \Phi_{J}$ may be interpreted as low-pass filter. $T_{J}$ is the convolution operator of this low-pass filter. Accordingly we understand the scale space $\mathcal{V}_{J}$ to be the image of $\mathcal{H}$ under the operator $T_{J}$ :

$$
\mathcal{V}_{J}=T_{J}(\mathcal{H})=\left\{\left(\Phi_{J} *_{\mathcal{H}} \Phi_{J}\right) *_{\mathcal{H}} F \mid F \in \mathcal{H}\right\}
$$

As an immediate consequence we obtain the following result.

Theorem 1.6. The scale spaces satisfy the following properties:

$$
\begin{aligned}
& \text { (i) }\left\{U_{0}^{*}\right\} \subset \mathcal{V}_{J} \subset \mathcal{V}_{J^{\prime}} \subset \mathcal{H}, \quad J \leq J^{\prime}, \\
& \text { (ii) } \bigcap_{J=-\infty}^{\infty} \mathcal{V}_{J}=\left\{U_{0}^{*}\right\} \\
& \text { (iii) } \bigcup_{J=-\infty}^{\infty} \mathcal{V}_{J} \cdot \|_{\mathcal{H}}=\mathcal{H}, \\
& \text { (iv) if } F_{J} \in \mathcal{V}_{J} \text { then } D_{-1} F_{J} \in \mathcal{V}_{J-1}, \quad J \in \mathbb{Z} .
\end{aligned}
$$

Proof. From the conditions (ii) and (iv) of Definition 1.4 we easily get the validity of the first assertion of Theorem 1.6. The identity (18) follows directly from the conditions (iii) and (iv) of Definition 1.4. The formula (19) is a consequence of Theorem 1.5, while (20) follows immediately from the definition of the shifting operator $D_{J}$. 
If a collection of subspaces of $\mathcal{H}$ satisfies the conditions of Theorem 1.6 we call them a multiresolution analysis (MRA).

The definition of the scaling functions now allows us to introduce $\mathcal{H}$-wavelets. Basic tool again is the concept of $\mathcal{H}$-kernels.

\section{$1.3 \quad \mathcal{H}$-wavelets}

We start with the definition of wavelets by aid of a "refinement (scaling) equation".

Definition 1.7. Let $\left\{\left(\Phi_{J}\right)^{\wedge}(n)\right\}_{n=0,1, \ldots}, J \in \mathbb{Z}$, be the generating symbol of an $\mathcal{H}$-scaling function as defined by Definition 1.4. Then the generating symbol $\left\{\left(\Psi_{j}\right)^{\wedge}(n)\right\}_{n=0,1, \ldots}, j \in \mathbb{Z}$, of the associated $\mathcal{H}$-wavelet is defined via the "refinement equation"

$$
\left(\Psi_{j}\right)^{\wedge}(n)=\left(\left(\left(\Phi_{j+1}\right)^{\wedge}(n)\right)^{2}-\left(\left(\Phi_{j}\right)^{\wedge}(n)\right)^{2}\right)^{\frac{1}{2}} .
$$

The family $\left\{\Psi_{j}\right\}, j \in \mathbb{Z}$, of $\mathcal{H}$-kernels given by

$$
\Psi_{j}(x, y)=\sum_{n=0}^{\infty}\left(\Psi_{j}\right)^{\wedge}(n) U_{n}^{*}(x) U_{n}^{*}(y), \quad x, y \in \Sigma,
$$

is called $\mathcal{H}$-wavelet associated to the $\mathcal{H}$-scaling function $\left\{\Phi_{J}\right\}, J \in \mathbb{Z}$. The corresponding mother wavelet is denoted by $\Psi_{0}$.

Clearly we are able to define a dilation and a shifting operator in the same way as we did before. For this reason any wavelet can be interpreted as a dilated and shifted copy of the corresponding mother wavelet like $\Psi_{j}(x, \cdot)=S_{x} D_{j} \Psi_{0}(\cdot, \cdot)$. We can easily derive from (21) that

$$
\begin{aligned}
\left(\left(\Phi_{J+1}\right)^{\wedge}(n)\right)^{2} & =\sum_{j=-\infty}^{J}\left(\left(\Psi_{j}\right)^{\wedge}(n)\right)^{2} \\
& =\left(\left(\Phi_{0}\right)^{\wedge}(n)\right)^{2}+\sum_{j=0}^{J}\left(\left(\Psi_{j}\right)^{\wedge}(n)\right)^{2} .
\end{aligned}
$$

Similar to the definition of the operator $T_{J}$ we are now led to convolution operators $R_{j}: \mathcal{H} \rightarrow \mathcal{H}$, given by

$$
R_{j} F=\left(\Psi_{j} *_{\mathcal{H}} \Psi_{j}\right) *_{\mathcal{H}} F, \quad F \in \mathcal{H} .
$$


Thus the identity

$$
\begin{aligned}
\Phi_{J+1} *_{\mathcal{H}} \Phi_{J+1} & =\sum_{j=-\infty}^{J}\left(\Psi_{j} *_{\mathcal{H}} \Psi_{j}\right) \\
& =\Phi_{0} *_{\mathcal{H}} \Phi_{0}+\sum_{j=0}^{J}\left(\Psi_{j} *_{\mathcal{H}} \Psi_{j}\right)
\end{aligned}
$$

can be written in operator formulation as follows:

$$
T_{J+1}=\sum_{j=-\infty}^{J} R_{j}=T_{0}+\sum_{j=0}^{J} R_{j} .
$$

The convolution operators $R_{j}$ describe the "detail information" of $F$ at scale $j$. In terms of filtering, $\left(\Psi_{j} *_{\mathcal{H}} \Psi_{j}\right), j \in \mathbb{Z}$, may be interpreted as a band-pass filter. This fact immediately gives rise to introduce the detail spaces as follows:

$$
\mathcal{W}_{j}=R_{j}(\mathcal{H})=\left\{\left(\Psi_{j} *_{\mathcal{H}} \Psi_{j}\right) *_{\mathcal{H}} F \mid F \in \mathcal{H}\right\}
$$

$\mathcal{W}_{J}$ contains the "detail information" needed to go from an approximation at level $J$ to an approximation at level $J+1$. Hence we get

$$
\sum_{j=-\infty}^{J} \mathcal{W}_{j}=\mathcal{V}_{0}+\sum_{j=0}^{J} \mathcal{W}_{j}=\mathcal{V}_{J+1}
$$

and

$$
\mathcal{V}_{J}+\mathcal{W}_{J}=\mathcal{V}_{J+1}, \quad J \in \mathbb{Z}
$$

It should be noted that, in general, the sum in (29) is neither direct nor orthogonal. But there exist examples leading to an orthogonal multiresolution which should be discussed later on.

In conclusion, any $F \in \mathcal{H}$ can be approximated as follows: starting with $T_{0} F$ we find (in connection to (27))

$$
T_{J+1} F=T_{0} F+\sum_{j=0}^{J} R_{j} F
$$

for any $J \in \mathbb{Z}$. In other words, the partial "reconstruction" $R_{J} F$ is nothing else than the "difference of two smoothings" at two consecutive scales

$$
R_{J} F=T_{J+1} F-T_{J} F .
$$


Definition 1.8. The wavelet transform $W T$ at scale $j \in \mathbb{Z}$ and position $x \in \Sigma$ is given by

$$
W T(F)(j ; x)=\left(\Psi_{j}(x, \cdot), F\right)_{\mathcal{H}}, \quad F \in \mathcal{H}
$$

Combining (33) and (26) we can formulate the main result of our wavelet theory as follows.

Theorem 1.9. Let $\left\{\left(\Phi_{J}\right)^{\wedge}(n)\right\}_{n=0,1, \ldots}, J \in \mathbb{Z}$, be the generating symbol of an $\mathcal{H}$-scaling function. Suppose that $\left\{\left(\Psi_{j}\right)^{\wedge}(n)\right\}_{n=0,1, \ldots}, j \in \mathbb{Z}$, is the generating symbol of the corresponding $\mathcal{H}$-wavelet. Furthermore, let $F$ be of class $\mathcal{H}$. Then

$$
F_{J}=\left(\Phi_{0} * \mathcal{H} \Phi_{0}\right) * \mathcal{H} F+\sum_{j=0}^{J-1}\left(\Psi_{j} * \mathcal{H} W T(F)(j, \cdot)\right)
$$

is the J-level approximation of $F$ satisfying

$$
\lim _{J \rightarrow \infty}\left\|F_{J}-F\right\|_{\mathcal{H}}=0 .
$$

The limit relation (35) shows the essential characteristic of wavelets. We change the approximated solution from $F_{J}$ to $F_{J+1}$ by adding the so-called detail information of level $J$ as the difference of two smoothings of two consecutive scales $J$ and $J+1$ and what is more important, we are able to guarantee $\lim _{J \rightarrow \infty} F_{J}=F$ in the sense of the $\|\cdot\|_{\mathcal{H}^{-}}$topology provided that $F \in \mathcal{H}$.

The following scheme summarizes the essential steps of our wavelet approach in the framework of a separable functional Hilbert space.

$$
\begin{aligned}
& T_{0}(G) \quad T_{1}(G) \ldots \quad T_{j}(G) \quad T_{j+1}(G) \ldots \stackrel{j \rightarrow \infty}{\rightarrow} F \\
& \widehat{\mathcal{V}}_{0} \subset \widehat{\mathcal{V}}_{1} \ldots \subset \subset \quad \widehat{\mathcal{V}}_{j} \subset \widehat{\mathcal{V}}_{j+1} \ldots=\stackrel{\Uparrow}{\mathcal{H}} \\
& \mathcal{V}_{0}+\mathcal{W}_{0}+\ldots+\mathcal{W}_{j-1}+\mathcal{W}_{j}+\ldots=\mathcal{H}
\end{aligned}
$$

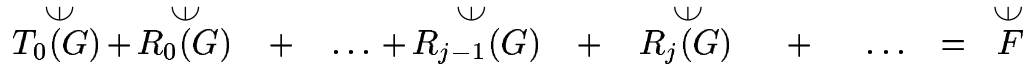

\section{Fast Multiscale Evaluation}

Until now efforts have been made to establish the basis property and the ability of bandpass filtering in terms of wavelets. Next we come to the third feature of 
wavelet approximation, viz. fast computation, which will be realized in form of a pyramid scheme for bandlimited wavelets.

For simplicity we assume that $\left\{\Phi_{j}(\cdot, \cdot)\right\}_{j \in \mathbb{Z}}$ is a family of bandlimited kernels, such that $\left(\left(\Phi_{j}\right)^{\wedge}(n)\right)^{2}>0$ for $n=0, \ldots, 2^{j}-1$ and $\left(\left(\Phi_{j}\right)^{\wedge}(n)\right)^{2}=0$ for $n \geq 2^{j}$. Then

$$
\Phi_{j}(x, \cdot) \in \mathcal{H}_{0, \ldots, 2^{j}-1}=\operatorname{span}\left\{U_{0}^{*}, \ldots, U_{2^{j}-1}^{*}\right\}
$$

and

$$
\Psi_{j}(x, \cdot) \in \mathcal{H}_{0, \ldots, 2^{j+1}-1}=\operatorname{span}\left\{U_{0}^{*}, \ldots, U_{2^{j+1}-1}^{*}\right\}
$$

holds for all $x \in \Sigma$, i.e.

$$
\begin{aligned}
& \Phi_{j}(x, y)=\sum_{n=0}^{2^{j}-1}\left(\Phi_{j}\right)^{\wedge}(n) U_{n}^{*}(x) U_{n}^{*}(y), \\
& \Psi_{j}(x, y)=\sum_{n=0}^{2^{j+1}-1}\left(\Psi_{j}\right)^{\wedge}(n) U_{n}^{*}(x) U_{n}^{*}(y)
\end{aligned}
$$

for $(x, y) \in \Sigma \times \Sigma$. Consequently, the scale spaces and the detail spaces, respectively, fulfill the relations $\mathcal{V}_{j}=\mathcal{H}_{0, \ldots, 2^{j}-1}, \mathcal{W}_{j} \subset \mathcal{H}_{0, \ldots, 2^{j+1}-1}$. Simple examples are given below:

(a) orthogonal (Shannon)

$$
\left(\Phi_{j}\right)^{\wedge}(n)=\left\{\begin{array}{ccc}
1 & \text { for } & n=0, \ldots, N_{j} \\
0 & \text { for } & n \geq N_{j}+1
\end{array},\right.
$$

(b) non-orthogonal (smoothed Shannon)

$$
\left(\Phi_{j}\right)^{\wedge}(n)=\left\{\begin{array}{rcc}
1 & \text { for } & n=0, \ldots, 2^{j} h \\
\frac{1-2^{-j} n}{1-h} & \text { for } & n=2^{j} h, \ldots, N_{j} \\
0 & \text { for } & n \geq N_{j}+1
\end{array}\right.
$$

for fixed $h \in[0,1)$,

(c) non-orthogonal (CP)

$$
\left(\Phi_{j}\right)^{\wedge}(n)=\left\{\begin{array}{rlc}
\left(1-2^{-j} n\right)^{2}\left(1+2^{-j+1} n\right) & \text { for } & n=0, \ldots, N_{j} \\
0 & \text { for } & n \geq N_{j}+1
\end{array}\right.
$$


with

$$
N_{j}=\left\{\begin{array}{rll}
0 & \text { for } & j \in \mathbb{Z}, j<0 \\
2^{j}-1 & \text { for } & j \in \mathbb{Z}, j \geq 0
\end{array} .\right.
$$

Note that the case (a) leads to an orthogonal multiresolution analysis, i.e. the detail and the scale spaces satisfy $\mathcal{V}_{j+1}=\mathcal{V}_{j} \oplus \mathcal{W}_{j}, \mathcal{W}_{j} \perp \mathcal{W}_{k}, k \neq j, j \geq 0$. In the cases (b) and (c) the scale and detail spaces are still finite-dimensional, but the detail spaces are no longer orthogonal.

Each scale space $\mathcal{V}_{j}, j \in \mathbb{N}_{0}$, can be understood as finite dimensional reproducing Hilbert space with the inner product $(\cdot, \cdot) \mathcal{V}_{j}$ and the reproducing kernel $\Gamma_{\mathcal{V}_{j}}$ being canonically defined by

$$
(F, G) \mathcal{V}_{j}=\sum_{n=0}^{N_{j}} \frac{1}{\left(\left(\Phi_{j}\right)^{\wedge}(n)\right)^{2}} F^{\wedge}(n) G^{\wedge}(n), \quad F, G \in \mathcal{H}_{0, \ldots, N_{j}}
$$

and

$$
\Gamma_{\mathcal{V}_{j}}(x, y)=\sum_{n=0}^{N_{j}}\left(\left(\Phi_{j}\right)^{\wedge}(n)\right)^{2} U_{n}^{*}(x) U_{n}^{*}(y), \quad x, y \in \Sigma,
$$

respectively. We observe that $\Gamma_{\mathcal{V}_{j}}=\Phi_{j} * \mathcal{H} \Phi_{j}=\Phi_{j}^{(2)}$ for all $j \in \mathbb{N}_{0}$.

The key idea of our fast evaluation method is based on the following observations:

(1) For some suitably large $J$, the scale space $\mathcal{V}_{J}$ is 'sufficiently close' to $\mathcal{H}$. Consequently, for each $F \in \mathcal{H}$, there exists a function of class $V_{J}$ such that the error between $F$ and $\Phi_{J}^{(2)} *_{\mathcal{H}} F$ (understood in the $\|\cdot\|_{\mathcal{H}}$-topology) is negligible. This is the reason why $F$ is assumed to be of class $\mathcal{V}_{J}$ for the remainder of this section.

(2) For $j=0, \ldots, J$ consider sequences $Y_{L_{j}}=\left\{y_{1}^{L_{j}}, \ldots, y_{L_{j}}^{L_{j}}\right\}, L_{j} \geq N_{j}$ of points $y_{i}^{L_{j}} \in \Sigma, i=1, \ldots, L_{j}, j=0,1, \ldots, J$, such that

$$
\mathcal{V}_{j}=\mathcal{H}_{0, \ldots, N_{j}}=\operatorname{span}\left(\Phi_{j}^{(2)}\left(\cdot, y_{1}^{L_{j}}\right), \ldots, \Phi_{j}^{(2)}\left(\cdot, y_{L_{j}}^{L_{j}}\right)\right)
$$

(the existence of pointsets $Y_{L_{j}} \subset \Sigma$ fulfilling the desired property is well-known from interpolation theory (see, for example, [2]). 
In an a priori step the coefficients $w_{l, k}^{L_{j}}$ have to be determined from the systems of linear equations

$$
\sum_{l=1}^{L_{j}} w_{l, k}^{L_{j}} \Phi_{j}^{(2)}\left(y_{i}^{L_{j}}, y_{l}^{L_{j}}\right)=\delta_{i, k}, \quad i, k=1, \ldots, L_{j},
$$

$j=0, \ldots, J$ and can be stored elsewhere. Looking carefully at the systems, it can be recognized that the coefficients $w_{l, k}^{L_{j}}$ do not depend on the particular function $F$ under consideration, but only on the chosen pointsystems.

Next our considerations are divided into two parts, viz. the initial step concerning the scale level $J$ and the pyramid step establishing the recursion relation.

\subsection{The Initial Step}

The exact approximation

$$
J_{L_{J}} S=\sum_{i=1}^{L_{J}} a_{i}^{L_{J}} S\left(y_{i}^{L_{J}}\right), \quad S \in \mathcal{V}_{J}
$$

to the bounded linear functional $\mathcal{L}$ on $\mathcal{V}_{J}$ defined by

$$
\mathcal{L} S=(S, F)_{\mathcal{V}_{J}}=S * \mathcal{V}_{J} F, \quad S \in \mathcal{V}_{J}, F \in \mathcal{V}_{J}
$$

is given by

$$
a_{i}^{L_{J}}=\sum_{k=1}^{L_{J}} w_{i, k}^{L_{J}} \mathcal{L} \Phi_{J}^{(2)}\left(\cdot, y_{k}^{L_{J}}\right), \quad i=1, \ldots, L_{J}
$$

Since $\Phi_{J}^{(2)}$ is the reproducing kernel of $\mathcal{V}_{J}$ (with respect to the $\|\cdot\|_{\mathcal{V}_{J}}$-topology), it follows in accordance with our assumption $F \in \mathcal{V}_{J}$, that $\Phi_{J}^{(2)} *_{\mathcal{V}_{J}} F=F$. Thus we find

$$
a_{i}^{L_{J}}=\sum_{k=1}^{L_{J}} w_{i, k}^{L_{J}}\left(\Phi_{J}^{(2)}\left(\cdot, y_{k}^{L_{J}}\right) * \mathcal{V}_{J} \quad F\right)=\sum_{k=1}^{L_{J}} w_{i, k}^{L_{J}} F\left(y_{k}^{L_{J}}\right)
$$

for $i=1, \ldots, L_{J}$.

This gives rise to the following conclusion. 
Lemma 2.1. If $F$ is a member of class $\mathcal{V}_{J}$, then

$$
S *_{\mathcal{V}_{J}} \quad F=\sum_{i=1}^{L_{J}} a_{i}^{L_{J}} S\left(y_{i}^{L_{J}}\right)
$$

holds for all $S \in \mathcal{V}_{J}$.

Lemma 2.1 immediately enables us to formulate

Lemma 2.2. Let $\Phi_{J}^{(2)} *_{\mathcal{H}} F$ be a function of class $\mathcal{V}_{J}$, then

$$
\Gamma *_{\mathcal{H}} \quad F=\sum_{i=1}^{L_{J}} a_{i}^{L_{J}} \Gamma\left(\cdot, y_{i}^{L_{J}}\right)
$$

holds for all $\mathcal{H}$-kernels $\Gamma(\cdot, \cdot)$ with $\Gamma^{\wedge}(n)=0$ for $n=N_{j}+1, N_{j}+2, \ldots$

Proof. $\Phi_{J}^{(2)} *_{\mathcal{H}} F \in \mathcal{V}_{J}$ can be represented as follows:

$$
\Phi_{J}^{(2)} *_{\mathcal{H}} \quad F=\sum_{i=1}^{L_{J}} a_{i}^{L_{J}} \Phi_{J}^{(2)}\left(\cdot, y_{i}^{L_{J}}\right) .
$$

In spectral language, i.e. expressed in terms of the orthonormal system $U_{n}^{*}$, this immediately implies

$$
F^{\wedge}(n)=\sum_{i=1}^{L_{J}} a_{i}^{L_{J}} U_{n}^{*}\left(y_{i}^{L_{J}}\right)
$$

for all $n=1, \ldots, N_{J}$. But this shows us that

$$
\begin{aligned}
\Gamma *_{\mathcal{H}} \quad F & =\sum_{n=0}^{\infty} \Gamma^{\wedge}(n) \sum_{i=1}^{L_{J}} a_{i}^{L_{J}} U_{n}^{*}\left(y_{i}^{L_{J}}\right) U_{n}^{*} \\
& =\sum_{i=1}^{L_{J}} a_{i}^{L_{J}} \Gamma\left(\cdot, y_{i}^{L_{J}}\right),
\end{aligned}
$$

as desired.

The next theorem clarifies the remarkable consequences for our wavelet concept. 
Theorem 2.3. Under the assumptions of Lemma 2.2 we have

$$
\begin{array}{rl}
\Phi_{J} * \mathcal{H} \quad F & =\sum_{i=1}^{L_{J}} a_{i}^{L_{J}} \Phi_{J}\left(\cdot, y_{i}^{L_{J}}\right) \\
\left(\Phi_{J} *_{\mathcal{H}} \Phi_{J}\right) *_{\mathcal{H}} & F=\sum_{i=1}^{L_{J}} a_{i}^{L_{J}}\left(\Phi_{J} *_{\mathcal{H}} \Phi_{J}\right)\left(\cdot, y_{i}^{L_{J}}\right)
\end{array}
$$

and

$$
\begin{aligned}
\Psi_{J-1} *_{\mathcal{H}} \quad F & =\sum_{i=1}^{L_{J}} a_{i}^{L_{J}} \Psi_{J-1}\left(\cdot, y_{i}^{L_{J}}\right) \\
\left(\Psi_{J-1} *_{\mathcal{H}}\right. & \left.\Psi_{J-1}\right) *_{\mathcal{H}} \quad F=\sum_{i=1}^{L_{J}} a_{i}^{L_{J}}\left(\Psi_{J-1} *_{\mathcal{H}} \Psi_{J-1}\right)\left(\cdot, y_{i}^{L_{J}}\right) .
\end{aligned}
$$

In conclusion, the vector $a^{L_{J}}=\left(a_{1}^{L_{J}}, \ldots, a_{L_{J}}^{L_{J}}\right)^{T} \in \mathbb{R}^{L_{J}}$ does $\underline{\text { not }}$ depend on the special choice of the $\Phi_{J}^{(2)}$-kernel in $\mathcal{V}_{J}$. Wavelet transform, lowpass, and bandpass filter can be computed by use of the same set of coefficients.

\subsection{The Pyramid Step}

The pyramid step provides an algorithm such that the vector $a^{L_{J}} \in \mathbb{R}^{L_{J}}$ serves as starting vector for a sequence of vectors $a^{L_{j}} \in \mathbb{R}^{L_{j}}, j=0, \ldots, J-1, J$, which fulfill the following properties:

(i) The vectors $a^{L_{j}}$ satisfy

$$
\Phi_{j}^{(2)} *_{\mathcal{H}} \quad F=\sum_{i=1}^{L_{j}} a_{i}^{L_{j}} \Phi_{j}^{(2)}\left(\cdot, y_{i}^{L_{j}}\right)
$$

for $j=0, \ldots, J$.

(ii) The wavelet transforms are given by

$$
\Psi_{j-1}^{(2)} *_{\mathcal{H}} \quad F=\sum_{i=1}^{L_{j}} a_{i}^{L_{j}} \Psi_{j-1}^{(2)}\left(\cdot, y_{i}^{L_{j}}\right)
$$

for $j=1, \ldots, J$. 
(iii) The vector $a^{L_{j}}$ is obtainable from $a^{L_{j+1}}, j=0, \ldots, J-1$, by recursion.

In the remainder of this section the properties (i), (ii) and (iii) are described in more detail.

The exact approximations $J_{L_{j}}, j=0, \ldots, J-1$,

$$
J_{L_{j}} S=\sum_{i=1}^{L_{j}} a_{i}^{L_{j}} S\left(y_{i}^{L_{j}}\right), \quad S \in \mathcal{V}_{j}
$$

to the bounded linear functional $\mathcal{L}$ on $\mathcal{V}_{j}$ defined by

$$
\mathcal{L} S=S *_{\mathcal{V}_{j}}\left(\Phi_{j}^{(2)} *_{\mathcal{H}} \quad F\right), \quad S \in \mathcal{V}_{j}, \quad F \in \mathcal{V}_{J},
$$

(note that $\Phi_{j}^{(2)} *_{\mathcal{H}} F \in \mathcal{V}_{j}$ ) are given by the coefficients

$$
a_{l}^{L_{j}}=\sum_{i=1}^{L_{j}} w_{l, i}^{L_{j}} \mathcal{L} \Phi_{j}^{(2)}\left(\cdot, y_{i}^{L_{j}}\right), \quad l=1, \ldots, L_{j} .
$$

Consequently we have for $l=1, \ldots, N_{j}$

$$
a_{l}^{L_{j}}=\sum_{i=1}^{L_{j}} w_{l, i}^{L_{j}}\left(\Phi_{j}^{(2)}\left(\cdot, y_{i}^{L_{j}}\right) * \mathcal{H} \quad F\right) .
$$

Thus we are led to the following lemma.

Lemma 2.4. If $\Phi_{j}^{(2)} *_{\mathcal{H}} \quad F$ is a member of class $\mathcal{V}_{j}$, then

$$
S * \mathcal{V}_{j}\left(\Phi_{j}^{(2)} * \mathcal{H} \quad F\right)=\sum_{i=1}^{L_{j}} a_{i}^{L_{j}} S\left(y_{i}^{L_{j}}\right)
$$

holds for all $S \in \mathcal{V}_{j}$.

By the same arguments as given in the last subsection we obtain

Lemma 2.5. Let $\Phi_{j}^{(2)} *_{\mathcal{H}} \quad F$ be a function of class $\mathcal{V}_{j}$, then

$$
\Gamma *_{\mathcal{H}} \quad F=\sum_{i=1}^{L_{j}} a_{i}^{L_{j}} \Gamma\left(\cdot, y_{i}^{L_{j}}\right)
$$

holds for all $\mathcal{H}$-kernels $\Gamma(\cdot, \cdot)$ with $\Gamma^{\wedge}(n)=0, n=N_{j}+1, N_{j}+2, \ldots$ (in particular for $\left.\Phi_{j}^{(2)}(\cdot, \cdot)\right)$. 
Finally this yields for the computation of the convolutions under consideration:

Theorem 2.6. Under the assumptions of Lemma 2.5 we have

$$
\begin{aligned}
\Phi_{j} *_{\mathcal{H}} F & =\sum_{i=1}^{L_{j}} a_{i}^{L_{j}} \Phi_{j}\left(\cdot, y_{i}^{L_{j}}\right), \\
\left(\Phi_{j} *_{\mathcal{H}} \Phi_{j} *_{\mathcal{H}}\right) F & =\sum_{i=1}^{L_{j}} a_{i}^{L_{j}}\left(\Phi_{j} *_{\mathcal{H}} \Phi_{j}\right)\left(\cdot, y_{i}^{L_{j}}\right)
\end{aligned}
$$

and

$$
\begin{aligned}
\Psi_{j-1} *_{\mathcal{H}} \quad F & =\sum_{i=1}^{L_{j}} a_{i}^{L_{j}} \Psi_{j-1}\left(\cdot, y_{i}^{L_{j}}\right) \\
\left(\Psi_{j-1} *_{\mathcal{H}} \quad \Psi_{j-1}\right) *_{\mathcal{H}} \quad F & =\sum_{i=1}^{L_{j}} a_{i}^{L_{j}}\left(\Psi_{j-1} *_{\mathcal{H}} \Psi_{j-1}\right)\left(\cdot, y_{i}^{L_{j}}\right) .
\end{aligned}
$$

From Theorem 2.6 we can deduce

$$
\Phi_{J-1}^{(2)} *_{\mathcal{H}} \quad F=\sum_{i=1}^{L_{J-1}} a_{i}^{L_{J-1}} \Phi_{J-1}^{(2)}\left(\cdot, y_{i}^{L_{J-1}}\right) .
$$

where

$$
a_{l}^{L_{J-1}}=\sum_{i=1}^{L_{J-1}} w_{l, i}^{L_{J-1}}\left(\Phi_{J-1}^{(2)}\left(\cdot, y_{i}^{L_{J-1}}\right) *_{\mathcal{H}} \quad F\right) .
$$

On the other hand, by virtue of Lemma 2.2 , we have

$$
\Phi_{J-1}^{(2)} *_{\mathcal{H}} \quad F=\sum_{i=1}^{L_{J}} a_{i}^{L_{J}} \Phi_{J-1}^{(2)}\left(\cdot, y_{i}^{L_{J}}\right)
$$

Combining (46) and (47) we obtain

$$
a_{l}^{L_{J-1}}=\sum_{i=1}^{L_{J-1}} \sum_{k=1}^{L_{J}} w_{l, i}^{L_{J-1}} a_{k}^{L_{J}} \Phi_{J-1}^{(2)}\left(y_{i}^{L_{J-1}}, y_{k}^{L_{J}}\right)
$$

for $l=1, \ldots, L_{J-1}$. If we assume the pointsets $Y_{L_{j}}$ to be hierarchical, i.e. $y_{i}^{L_{j}}=y_{i}^{L_{j+1}}, i=1, \ldots, L_{j}, j=0, \ldots, J-1$ and observing the symmetry of 
the matrix $\left(w_{l, i}^{L_{J-1}}\right)$, we are led to a reduction of computational costs as follows:

$$
\begin{aligned}
a_{l}^{L_{J-1}} & =\sum_{i=1}^{L_{J-1}} \sum_{k=1}^{L_{J}} w_{i, l}^{L_{J-1}} a_{k}^{L_{J}} \Phi_{J-1}^{(2)}\left(y_{i}^{L_{J-1}}, y_{k}^{L_{J}}\right) \\
& =\sum_{i=1}^{L_{J-1}} \sum_{k=1}^{L_{J-1}} w_{i, l}^{L_{J-1}} a_{k}^{L_{J}} \Phi_{J-1}^{(2)}\left(y_{i}^{L_{J-1}}, y_{k}^{L_{J-1}}\right) \\
& +\sum_{i=1}^{L_{J-1}} \sum_{k=L_{J-1}+1}^{L_{J}} w_{i, l}^{L_{J-1}} a_{k}^{L_{J}} \Phi_{J-1}^{(2)}\left(y_{i}^{L_{J-1}}, y_{k}^{L_{J}}\right) \\
& =a_{l}^{L_{J}}+\sum_{i=1}^{L_{J-1}} \sum_{k=L_{J-1}+1}^{L_{J}} w_{i, l}^{L_{J-1}} a_{k}^{L_{J}} \Phi_{J-1}^{(2)}\left(y_{i}^{L_{J-1}}, y_{k}^{L_{J}}\right) .
\end{aligned}
$$

The recursion relation (48) leads us to the following decomposition scheme:

$$
\begin{aligned}
& F \quad \rightarrow \quad a^{L_{J}} \quad \rightarrow \quad a^{L_{J-1}} \quad \rightarrow \quad \ldots \quad a^{L_{0}} \\
& \begin{array}{ccc}
\stackrel{\downarrow}{\downarrow} & \stackrel{\downarrow}{L_{J}} & \stackrel{\downarrow}{ } \\
(W T)(F)(J ; \cdot) & (W T)(F)(J-1 ; \cdot) & (W T)(F)(0 ; \cdot)
\end{array}
\end{aligned}
$$

The bandpass filter $R_{j}(F)$ can be deduced from the formula

$$
\begin{aligned}
R_{j}(F) & =\Psi_{j} * \mathcal{H}(W T)(F)(j ; \cdot) \\
& =\sum_{i=1}^{L_{j}} a_{i}^{L_{j}}\left(\begin{array}{lll}
\Psi_{j} & * \mathcal{H} & \Psi_{j}
\end{array}\right)\left(y_{i}^{L_{j}}, \cdot\right) .
\end{aligned}
$$

This allows the following reconstruction scheme of $F$ :

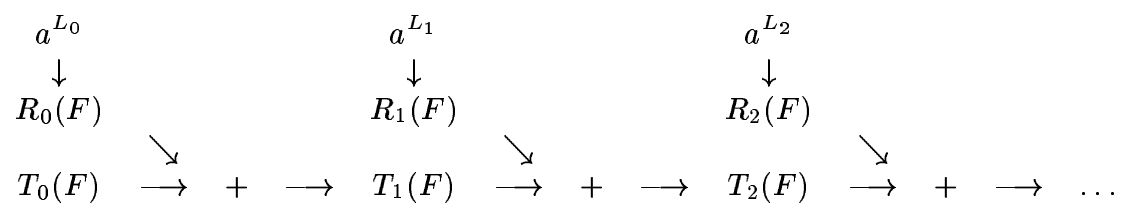

We have seen that the vectors $a^{L_{j}}$ do not depend on the special choice of the scaling function $\left\{\Phi_{j}\right\}_{j \in \mathbb{N}_{0}}$. In other words, we are able to reconstruct a function with respect to different wavelets just by the knowledge of the vectors $a^{L_{j}}$. 


\section{Gravitational Field Determination}

In this section we discuss as a georelevant example the problem of multiscale gravitational field determination using single- and multipoles. For that purpose we understand $\Sigma$ to be the surface of the earth which is assumed to be regular (i.e.: (i) $\Sigma$ divides $\mathbb{R}^{3}$ uniquely into the bounded region $\Sigma^{\text {int }}$ (inner space) and the unbounded region $\Sigma^{e x t}$ (outer space) determined by $\Sigma^{e x t}=\mathbb{R}^{3} \backslash \overline{\Sigma^{i n t}}$, $\overline{\Sigma^{i n t}}=\Sigma \cup \Sigma^{i n t}$, (ii) $\Sigma^{i n t}$ contains the origin, (iii) $\Sigma$ is a closed and compact $C^{(2)}$-surface being free of double points). As trial systems $\left\{U_{n}\right\}_{n=0,1, \ldots}$ for gravitational field determination we use the following examples (cf. [1]):

(i) single poles

$$
\frac{1}{\left|x-x_{n}\right|}, \quad n=0,1, \ldots
$$

where $\left(x_{n}\right)_{n=0,1, \ldots}$ forms a pointset which is dense on a regular surface $B$ inside the earth (for instance, $B$ may be chosen as a "Bjerhammar sphere" or a surface parallel to $\Sigma$ in the earth's interior).

(ii) multipoles

$$
\left(\frac{\partial}{\partial x_{0}}\right)^{j} \frac{1}{\left|x-x_{0}\right|}, \quad[j]=n, n=0,1, \ldots
$$

where $x_{0}$ is a fixed point in $\Sigma^{i n t}, j=\left(j_{1}, j_{2}, j_{3}\right)$ is a triple of non-negative integers with

$$
[j]=j_{1}+j_{2}+j_{3},\left(\frac{\partial}{\partial x_{0}}\right)^{j}=\left.\frac{\partial^{[j]}}{\partial x_{1}^{j_{1}} \partial x_{2}^{j_{2}} \partial x_{3}^{j_{3}}}\right|_{x=x_{0}}
$$

(for instance, $x_{0}$ can be chosen to be the origin).

(iii) solid spherical harmonics

$$
\left(\frac{\alpha}{|x|}\right)^{n+1} Y_{n, k}\left(\frac{x}{|x|}\right), \quad n=0,1, \ldots ; k=1, \ldots, 2 n+1,
$$

where $\alpha$ is a radius satisfying $\alpha<i n f_{x \in \Sigma}|x| .\left\{Y_{n, k}\right\}_{k=1, \ldots, 2 n+1}$ denotes a (maximal) linearly independent system of spherical harmonics of degree $n, n=0,1, \ldots$ 
(iv) solid ellipsoidal harmonics

$$
\frac{Q_{n, k}\left(i \frac{u}{E}\right)}{Q_{n, k}\left(i \frac{b}{E}\right)} Y_{n, k}\left(\frac{x}{|x|}\right), \quad n=0,1, \ldots, k=1, \ldots, 2 n+1,
$$

where $Q_{n, k}$ are the Legendre functions of second kind, $x$ is an element of an ellipsoid of revolution with semiminor axis $u$ and $b$ denotes the semiminor axis of an arbitrary but fixed (internal) ellipsoid which may be called the reference ellipsoid (cf. [3]). If the eccentricity $E$ reduces to zero, then the reference ellipsoid becomes the sphere with radius $\alpha$ and the fraction $Q_{n, k}(i u / E) / Q_{n, k}(i b / E)$ reduces to $(\alpha /|x|)^{n+1}$.

(v) Dirichlet (evaluation) functionals

$$
\Gamma\left(x, x_{n}\right), \quad n=0,1, \ldots,
$$

where $\left(x_{n}\right)_{n=0,1, \ldots}$ forms a countable dense system of points on $\Sigma$ and $\Gamma$ induced by one of the trial systems (i)-(iv) denotes an $\mathcal{H}$-kernel with $\mathcal{H}$-admissible symbol $\Gamma^{\wedge}(n)$.

Then it is known from [1] for our trial systems that

$$
\mathcal{L}^{2}(\Sigma)=\overline{\operatorname{span}_{n=0,1, \ldots}\left(U_{n} \mid \Sigma\right)}\|\cdot\|_{\mathcal{L}^{2}(\Sigma)}
$$

and

$$
C(\Sigma)=\overline{\operatorname{span}_{n=0,1, \ldots}\left(\left.U_{n}\right|_{\Sigma}\right)} \mid \cdot \|_{C(\Sigma)}
$$

Moreover, we have

$$
\operatorname{Pot}^{(0)}\left(\overline{\bar{\Sigma}^{e x t}}\right)=\overline{\operatorname{span}_{n=0,1, \ldots}\left(\left.U_{n}\right|_{\overline{\Sigma^{e x t}}}\right)}\|\cdot\|_{C\left(\overline{\Sigma^{e x t}}\right)},
$$

where $\operatorname{Pot}^{(0)}\left(\overline{\Sigma^{e x t}}\right)$ is the space of all $V \in C^{(2)}\left(\Sigma^{e x t}\right) \cap C^{(0)}\left(\overline{\Sigma^{e x t}}\right)$ satisfying $\Delta V=0$ in $\Sigma^{e x t}$ and $V(x)=O(1 /|x|),|x| \rightarrow \infty$. In order to make use of our Hilbert space framework of wavelets we have to orthonormalize the system $\left(U_{n}\right)_{n=0,1, \ldots}$ (for example, by virtue of the well-known Gram-Schmidt process) with respect to the $\|\cdot\|_{\mathcal{L}^{2}(\Sigma)}$-topology obtaining a system $\left(H_{n}^{*}\right)_{n=0,1, \ldots}$, $\left(H_{n}^{*}\right) \in \operatorname{Pot}^{(0)}\left(\overline{\Sigma^{e x t}}\right), n=0,1, \ldots$ with the following properties:

(i) $\left(U_{n}^{*}\right)_{n=0,1, \ldots}$ defined by $U_{n}^{*}=\left.H_{n}^{*}\right|_{\Sigma}$ is a complete orthonormal system in the Hilbert space $\left(\left(\mathcal{L}^{2}(\Sigma),(\cdot, \cdot)\right)_{\mathcal{L}^{2}(\Sigma)}\right)$ 
(ii) $H_{n}^{*}$ is the unique solution of the boundary value problem $H_{n}^{*} \in P o t^{(0)}\left(\overline{\Sigma^{e x t}}\right)$ corresponding to the Dirichlet data $\left.H_{n}^{*}\right|_{\Sigma}=U_{n}^{*}, n=0,1, \ldots$, respectively.

Let $V$ be the uniquely determined solution of the boundary value problem $V \in \operatorname{Pot}^{(0)}\left(\overline{\Sigma^{e x t}}\right),\left.V\right|_{\Sigma}=F$. Then it is known (cf. [1]) for our trial system that the $\mathcal{L}^{2}(\Sigma)$-convergence of the Fourier expansion

$$
F_{N}=\sum_{n=0}^{N} F^{\wedge}(n) U_{n}^{*}
$$

with

$$
F^{\wedge}(n)=\left(F, U_{n}^{*}\right)_{\mathcal{L}^{2}(\Sigma)}=\int_{\Sigma} V(x) U_{n}^{*}(x) d \omega_{\Sigma}(x)
$$

to the function $F$ on the surface $\Sigma$ implies ordinary pointwise convergence of the sum

$$
V_{N}=\sum_{n=0}^{N} F^{\wedge}(n) H_{n}^{*}
$$

to the potential $V$ as $N \rightarrow \infty$ for every point $x \in K$ with $K \subset \Sigma^{e x t}$ and $\operatorname{dist}(K, \Sigma) \geq \rho>0$. In every compact subset $K \subset \Sigma^{e x t}$ the convergence is uniform (cf. [1]). To be more specific, the limit relation

$$
\lim _{N \rightarrow \infty}\left\|F-F_{N}\right\|_{\mathcal{L}^{2}(\Sigma)}=0
$$

implies

$$
\lim _{N \rightarrow \infty} \sup _{x \in K}\left|V(x)-V_{N}(x)\right|=0 .
$$

From our wavelet approach presented above it follows that the $J$-level wavelet approximation $F_{J}$ given by

$$
\begin{aligned}
F_{J} & =\left(\Phi_{0}^{\left(U_{n}^{*}\right)} *_{\mathcal{L}^{2}(\Sigma)} \Phi_{0}^{\left(U_{n}^{*}\right)}\right) *_{\mathcal{L}^{2}(\Sigma)} F \\
& +\sum_{j=0}^{J-1}\left(\Psi_{j}^{\left(U_{n}^{*}\right)} *_{\mathcal{L}^{2}(\Sigma)} \Psi_{j}^{\left(U_{n}^{*}\right)}\right) *_{\mathcal{L}^{2}(\Sigma)} F
\end{aligned}
$$


converges to the function $F$ in $\|\cdot\|_{\mathcal{L}^{2}(\Sigma)}$-norm, where we have used the canonical abbreviations

$$
\begin{aligned}
& \Phi_{j}^{\left(U_{n}^{*}\right)}(x, y)=\sum_{n=0}^{\infty}\left(\Phi_{j}\right)^{\wedge}(n) U_{n}^{*}(x) U_{n}^{*}(y) \\
& \Psi_{j}^{\left(U_{n}^{*}\right)}(x, y)=\sum_{n=0}^{\infty}\left(\Psi_{j}\right)^{\wedge}(n) U_{n}^{*}(x) U_{n}^{*}(y)
\end{aligned}
$$

for $j \in \mathbb{Z}$ and $(x, y) \in \Sigma \times \Sigma$. But this immediately implies that

$$
\begin{aligned}
V_{J} & =\left(\Phi_{0}^{\left(H_{n}^{*}\right)} *_{\mathcal{L}^{2}(\Sigma)} \Phi_{0}^{\left(H_{n}^{*}\right)}\right) * \mathcal{L}^{2}(\Sigma) \\
& +\sum_{j=0}^{J-1}\left(\Psi_{j}^{\left(H_{n}^{*}\right)} *_{\mathcal{L}^{2}(\Sigma)} \Psi_{j}^{\left(H_{n}^{*}\right)}\right) *_{\mathcal{L}^{2}(\Sigma)} F
\end{aligned}
$$

with

$$
\begin{aligned}
& \Phi_{j}^{\left(H_{n}^{*}\right)}(x, y)=\sum_{n=0}^{\infty}\left(\Phi_{j}\right)^{\wedge}(n) H_{n}^{*}(x) H_{n}^{*}(y) \\
& \Psi_{j}^{\left(H_{n}^{*}\right)}(x, y)=\sum_{n=0}^{\infty}\left(\Psi_{j}\right)^{\wedge}(n) H_{n}^{*}(x) H_{n}^{*}(y)
\end{aligned}
$$

converges in pointwise sense to the potential $V \in \operatorname{Pot}^{(0)}\left(\overline{{\overline{\Sigma^{e x t}}}}\right)$ with $\left.V\right|_{\Sigma}=F$ as $J \rightarrow \infty$ for every $x \in K \subset \Sigma^{e x t}$ and $\operatorname{dist}(K, \Sigma) \geq \rho>0$. In every compact subset $K \subset \Sigma^{e x t}$ the convergence is in fact uniform, i.e.

$$
\lim _{J \rightarrow \infty} \sup _{x \in K}\left|V(x)-V_{J}(x)\right|=0
$$

for $J \in \mathbb{Z}$ and $(x, y) \in \overline{\Sigma^{e x t}} \times \overline{\Sigma^{e x t}}$.

Finally we give a numerical test example. In accordance with the EGM96model, we choose $\Sigma$ to be a spherical earth's surface, i.e. $\Sigma$ is supposed to be a sphere around the origin (centre of gravity) with mean earth's radius $R=6378 \mathrm{~km} . \quad V$ is the earth's gravitational potential corresponding to the EGM96 model on $\Sigma$. The multiresolution analysis 'looks at' the earth's gravitational potential through a microscope, whose resolution gets finer and finer. Thus it associates to the gravitational a sequence of smoothed versions, labelled by the scale parameter. An illustration of the gravitational potential as a multiresolution analysis at the earth's surface is shown for the levels $j=3, \ldots, 8$ based on the solid spherical harmonics as orthonormal system. The computations have been performed on the basis of the CP-wavelets. 

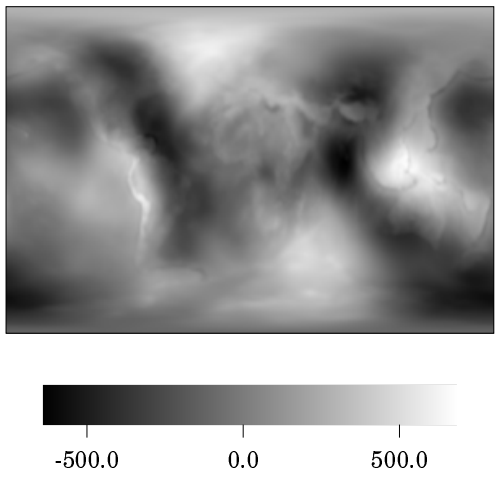

raw data set
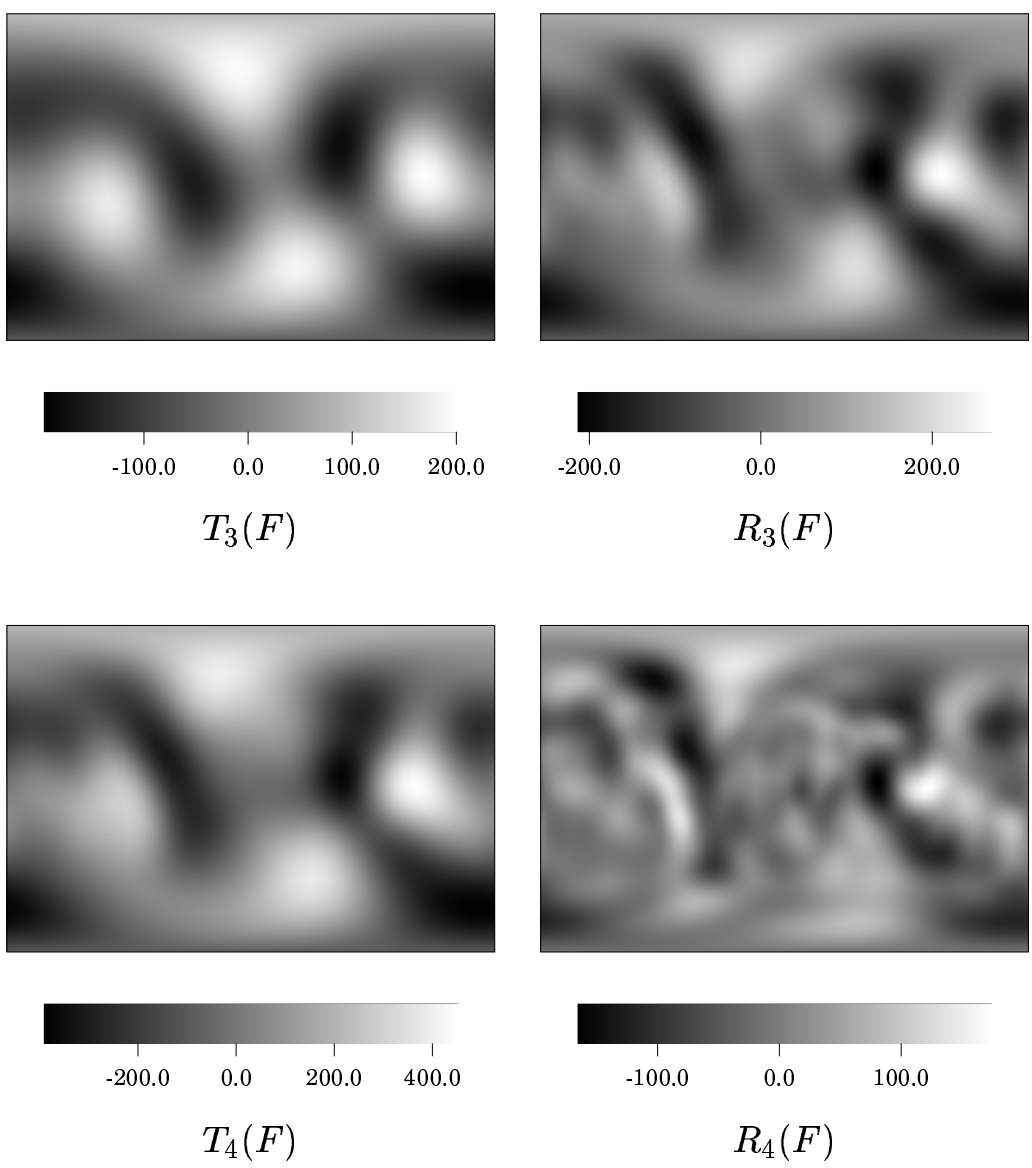

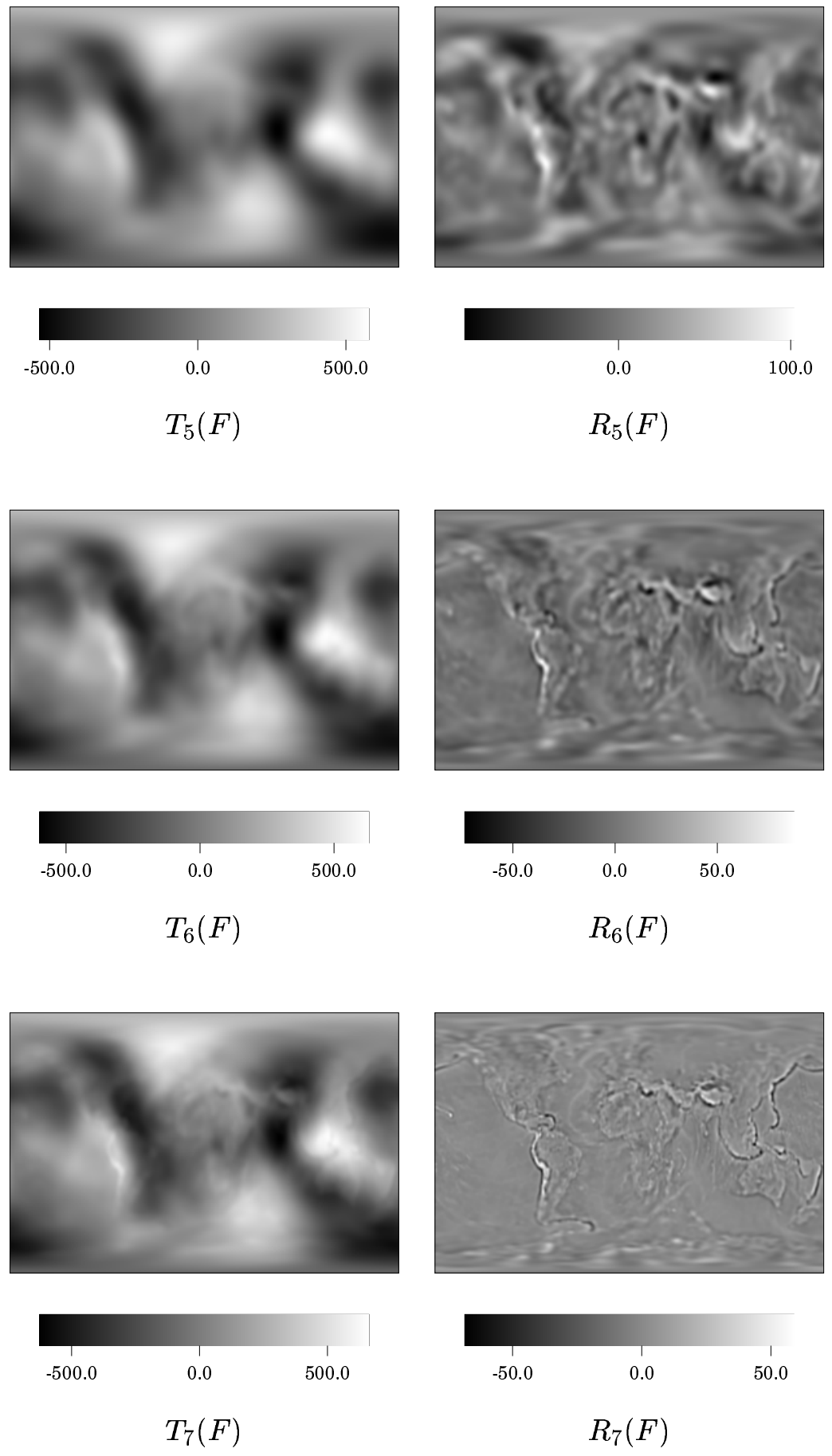


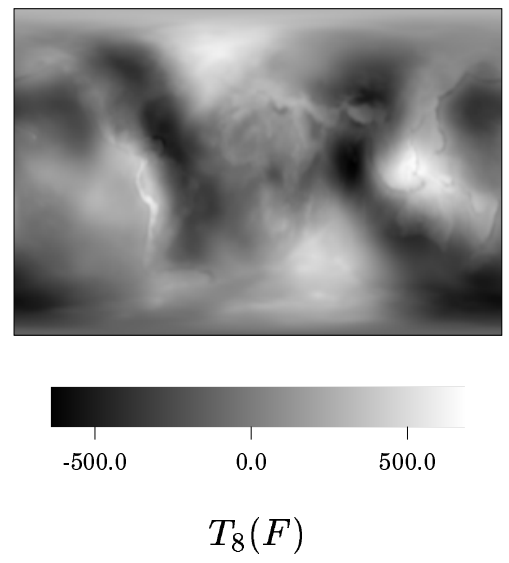

\section{References}

[1] W. Freeden. On the Approximation of External Gravitational Potential with Closed Systems of (Trial) Functions. Bull. Geod., 54:1-20, 1980.

[2] W. Freeden, T. Gervens, and M. Schreiner. Constructive Approximation on the Sphere (With Applications to Geomathematics). Oxford Science Publications, Clarendon, 1998.

[3] W.A. Heiskanen and H. Moritz. Physical Geodesy. W.H. Freeman and Company, 1967.

\section{Addresse:}

University of Kaiserslautern

Geomathematics Group

67653 Kaiserslautern

P.O. Box 3049

Germany

email: freeden@mathematik.uni-kl.de

www: http://www.mathematik.uni-kl.de/ wwwgeo 